Supporting Information for:

\title{
Empirical Valence Bond Simulations of the Hydride-Transfer Step in the Monoamine Oxidase A Catalyzed Metabolism of Noradrenaline
}

\author{
Matic Poberžnik, ${ }^{1}$ Miha Purg, ${ }^{2}$ Matej Repič, ${ }^{3}$ Janez Mavri, ${ }^{3, *}$ and Robert Vianello, ${ }^{4, *}$ \\ ${ }^{1}$ Department of Physical and Organic Chemistry, Jožef Stefan Institute, Jamova cesta 39, SI-1000 Ljubljana, \\ Slovenia. \\ ${ }^{2}$ Department of Cell and Molecular Biology, Uppsala Biomedical Centre, Husargatan 3, S-75124 Uppsala, \\ Sweden. \\ ${ }^{3}$ Laboratory for Biocomputing and Bioinformatics, National Institute of Chemistry, Hajdrihova ulica 19, SI-1000 \\ Ljubljana, Slovenia. Phone: +386 1 4760309. E-mail: janez.mavri@ki.si \\ ${ }^{4}$ Computational Organic Chemistry and Biochemistry Group, Ruđer Bošković Institute, Bijenička cesta 54, HR- \\ 10000 Zagreb, Croatia. Phone: +385 14561117. E-mail: robert.vianello@irb.hr
}

\section{Table of Contents}

- Determination of reliable experimental activation free energy for noradrenaline S2-S4 degradation within MAO A enzyme from the various values reported in the literature

- Parameterization charges of the FAD co-factor, lumiflavin moiety and noradrenaline molecule for use with the ENZYMIX force-field

- MD parameters for the non-standard residues in the EVB region as defined in the MOLARIS program package

- Figure S1. The initial placement of substrate in the studied mutated enzymes.

- References 


\section{Determination of reliable experimental activation free energy for noradrenaline}

\section{degradation within MAO A enzyme from the various values reported in the}

\section{literature}

The only available experimental rate constant for $R$-noradrenaline (levorotary (-)) with human MAO A (hMAO A) was published in 1985, at a time when recombinant MAO was unavailable and experimental rate constants were determined using homogenates of various tissues. ${ }^{1}$ The results of this work are reported as $V_{\max }$ values in pmol $\mathrm{mg}_{\text {protein }}{ }^{-1} \mathrm{~min}^{-1}$ units, which have to be converted to the $k_{\text {cat }}$ values in $\min ^{-1}$ units. Taking into account that the molecular mass of the human MAO A monomer (PDB accession code $2 \mathrm{Z} 5 \mathrm{X}$ ) is $59969.12 \mathrm{~g} \mathrm{~mol}^{-1}$, we can obtain the $k_{\text {cat }}$ using the following equation: $k_{\text {cat }}=V_{\text {max }}$. $M$ protein. Subsequently, we can convert the rate constant to the free energy of activation using the Eyring-Polanyi equation, obtaining the results in Table S1 and Table S2:

$$
\Delta G^{\ddagger}=-\mathrm{RT} \cdot \ln \left(V_{\text {max }} M_{\text {protein }} \frac{h}{k_{\mathrm{B}} T}\right)
$$

Table S1. Free energies of activation computed from the $V_{\max }$ values reported by O'Carroll an co-workers in ref. 1.

\begin{tabular}{|c|c|c|c|c|}
\hline Substrate & $V_{\max }\left[\mathrm{pmol} \mathrm{mg}^{-1} \min ^{-1}\right]$ & $k_{\text {cat }}\left[\min ^{-1}\right]$ & $\Delta G^{\ddagger}\left[\mathrm{kcal} \mathrm{mol}^{-1}\right]$ & $\operatorname{Temp}\left[{ }^{\circ} \mathrm{C}\right]$ \\
\hline dopamine & 680 & 0.041 & 22.66 & 37.0 \\
\hline phenylethylamine & 20 & 0.001 & 24.84 & 37.0 \\
\hline$R$-noradrenaline & 561 & 0.034 & 22.78 & 37.0 \\
\hline serotonin & 228 & 0.014 & 23.34 & 37.0 \\
\hline
\end{tabular}


Table S2. Free energies of activation $\left(\Delta G^{\ddagger}\right)$ computed from the $k_{\text {cat }}$ values reported for the wild-type human hMAO A enzyme.

\begin{tabular}{|c|c|c|c|c|c|}
\hline Substrate & $V_{\max }\left[p m o l \mathrm{mg}^{-1} \min ^{-1}\right]$ & $k_{\text {cat }}\left[\min ^{-1}\right]$ & $\Delta G^{\ddagger}\left[\mathrm{kcal} \mathrm{mol}^{-1}\right]$ & $\operatorname{Temp}\left[{ }^{\circ} \mathrm{C}\right]$ & Ref. \\
\hline dopamine & N/A & $71.1^{\mathrm{a}}$ & 16.51 & 11 & 2 \\
\hline dopamine & N/A & $81.5^{\mathrm{b}}$ & 16.43 & 11 & 2 \\
\hline phenylethylamine & $\mathrm{N} / \mathrm{A}$ & $64.0^{\mathrm{a}}$ & 16.57 & 11 & 3 \\
\hline phenylethylamine & N/A & $43.8^{\mathrm{a}}$ & 16.78 & 11 & 3 \\
\hline phenylethylamine & N/A & $64.0^{\mathrm{a}}$ & 16.57 & 11 & 2 \\
\hline phenylethylamine & N/A & $53.8^{\mathrm{b}}$ & 16.67 & 11 & 2 \\
\hline phenylethylamine & $\mathrm{N} / \mathrm{A}$ & $186.0^{\mathrm{b}}$ & 17.07 & 30 & 4 \\
\hline phenylethylamine & N/A & $53.8^{\mathrm{b}}$ & 17.52 & 25 & 5 \\
\hline phenylethylamine & $\mathrm{N} / \mathrm{A}$ & $53.8^{\mathrm{b}}$ & 17.52 & 25 & 6 \\
\hline phenylethylamine & $\mathrm{N} / \mathrm{A}$ & $59.9^{\mathrm{b}}$ & 17.45 & 25 & 6 \\
\hline serotonin & $\mathrm{N} / \mathrm{A}$ & $182.0^{\mathrm{a}}$ & 15.98 & 11 & 2 \\
\hline serotonin & $\mathrm{N} / \mathrm{A}$ & $175.1^{\mathrm{b}}$ & 16.00 & 11 & 2 \\
\hline serotonin & N/A & $1116.0^{b}$ & 15.99 & 30 & 4 \\
\hline serotonin & $\mathrm{N} / \mathrm{A}$ & $175.1^{b}$ & 16.82 & 25 & 5 \\
\hline serotonin & N/A & $198.0^{\mathrm{b}}$ & 16.74 & 25 & 7 \\
\hline serotonin & N/A & $183.0^{\mathrm{b}}$ & 16.79 & 25 & 7 \\
\hline
\end{tabular}

${ }^{a}$ hMAO A expressed from Saccharomyces cerevisiae. ${ }^{b}$ hMAO A expressed from Pichia pastoris.

By comparing values from Table S1 with values from Table S2, it is evident that the barriers listed in Table S1 are systematically too high in comparison with the more recent experimental values. Therefore, we decided to correlate the values for dopamine, phenylethylamine and serotonin from Table S1 with the average of the recent experimental values: 
Table S3. $\Delta G^{\ddagger}$ values from Table $\mathrm{S} 1$ and average $\Delta G^{\ddagger}$ values for dopamine, phenylethylamine and serotonin calculated from various experimental data collected in Table S2.

\begin{tabular}{|c|c|c|}
\hline Substrate & $\Delta G_{\text {s1 }}^{*}\left[\mathrm{kcal} \mathrm{mol}^{-1}\right]$ & Average $\Delta G_{\mathrm{s} 2}^{\dagger}\left[\mathrm{kcal} \mathrm{mol}^{-1}\right]$ \\
\hline dopamine & 22.66 & 16.47 \\
\hline phenylethylamine & 24.84 & 17.02 \\
\hline serotonin & 23.34 & 16.39 \\
\hline
\end{tabular}

Using linear regression we obtained the following equation $\Delta G_{\mathrm{S} 2}=0.28 \cdot \Delta G_{\mathrm{S} 1}+10.1$ with the regression coefficient $\mathrm{R}^{2}=0.83$. Since $\Delta G^{\star}$ S1 for $R$-noradrenaline is $22.78 \mathrm{kcal} \mathrm{mol}^{-1}$ (Table $\mathrm{S} 1$ ), the best estimate for the experimental barrier is $16.5 \mathrm{kcal} \mathrm{mol}^{-1}$ using the above mentioned equation. 
Parameterization charges of the FAD co-factor, lumiflavin moiety and noradrenaline molecule for use with the ENZYMIX force-field

Table S4. Noradrenaline (NA) charges. (The transferring hydrogen atom is indicated in shading)

\begin{tabular}{|c|ccc|ccc|} 
NA & \multicolumn{3}{|c|}{ Reactant State } & \multicolumn{3}{c|}{ Product State } \\
Atom ID & Atom Name & Initial (0 ps) & Final (60 ps) & Atom Name & Initial (0 ps) & Final (60ps) \\
\hline 1 & OAC & -0.63 & -0.56 & OAC & -0.63 & -0.57 \\
2 & CAJ & 0.46 & 0.41 & CAJ & 0.42 & 0.44 \\
3 & CAG & -0.45 & -0.43 & CAG & -0.41 & -0.44 \\
4 & CAI & 0.18 & 0.32 & CAI & 0.22 & 0.31 \\
5 & OAB & -0.60 & -0.57 & OAB & -0.60 & -0.54 \\
6 & CAE & -0.18 & -0.38 & CAE & -0.19 & -0.34 \\
7 & CAF & -0.22 & -0.17 & CAF & -0.22 & -0.23 \\
8 & CAK & -0.13 & -0.19 & CAK & -0.08 & -0.08 \\
9 & CAL & 0.52 & 0.40 & CAL & 0.42 & 0.40 \\
10 & OAD & -0.74 & -0.67 & OAD & -0.67 & -0.69 \\
11 & CAH & 0.30 & 0.26 & CAH & 0.25 & 0.31 \\
12 & NAA & -1.11 & -1.20 & NAA & -0.51 & -0.60 \\
13 & HAG & 0.21 & 0.16 & HAG & 0.22 & 0.21 \\
14 & HAE & 0.16 & 0.19 & HAE & 0.17 & 0.19 \\
15 & HAF & 0.16 & 0.16 & HAF & 0.17 & 0.18 \\
16 & HAL & -0.06 & -0.02 & HAL & 0.05 & 0.03 \\
17 & HAH2 & -0.01 & 0.03 & HAH2 & 0.20 & 0.16 \\
18 & HAH3 & $\mathbf{0 . 0 1}$ & $\mathbf{0 . 0 4}$ & & & 0.45 \\
19 & HAD & 0.46 & 0.41 & HAD & 0.48 & 0.43 \\
20 & HAC & 0.42 & 0.46 & HAC & 0.43 & 0.46 \\
21 & HAB & 0.43 & 0.46 & HAB & 0.43 & 0.46 \\
22 & HAA1 & 0.42 & 0.45 & HAA1 & 0.43 & 0.44 \\
23 & HAA2 & 0.40 & 0.44 & HAA2 & 0.42 & 0.45
\end{tabular}


Table S5. Flavin (FAD) charges. (The transferring hydrogen and the accepting N5 atom of the co-factor are indicated in shading)

\begin{tabular}{|c|c|c|c|c|c|c|}
\hline \multirow{2}{*}{$\begin{array}{c}\text { FAD } \\
\text { Atom ID }\end{array}$} & \multicolumn{3}{|c|}{ Reactant State } & \multicolumn{3}{|c|}{ Product State } \\
\hline & Atom Name & Initial (0 ps) & Final (60ps) & Atom Name & Initial (0ps) & Final $(60 \mathrm{ps})$ \\
\hline 1 & $\mathrm{CAG}$ & -0.47 & -0.51 & CAG & -0.52 & -0.51 \\
\hline 2 & $\mathrm{CG}$ & 0.74 & 0.74 & $\mathrm{CG}$ & 0.81 & 0.74 \\
\hline 3 & OG & -0.74 & -0.74 & OG & -0.68 & -0.74 \\
\hline 4 & HA1G & 0.15 & 0.17 & HA1G & 0.14 & 0.17 \\
\hline 5 & HA2G & 0.16 & 0.17 & $\mathrm{HA} 2 \mathrm{G}$ & 0.15 & 0.17 \\
\hline 6 & HA3G & 0.16 & 0.17 & HA3G & 0.15 & 0.17 \\
\hline 7 & $\mathrm{NC}$ & -0.49 & -0.42 & $\mathrm{NC}$ & -0.69 & -0.43 \\
\hline 8 & $\mathrm{CAC}$ & 0.00 & 0.00 & $\mathrm{CAC}$ & 0.28 & 0.00 \\
\hline 9 & $\mathrm{CC}$ & 0.57 & 0.57 & $\mathrm{CC}$ & 0.52 & 0.54 \\
\hline 10 & $\mathrm{OC}$ & -0.57 & -0.57 & $\mathrm{OC}$ & -0.63 & -0.54 \\
\hline 11 & $\mathrm{CBC}$ & -0.30 & -0.18 & $\mathrm{CBC}$ & -0.26 & -0.25 \\
\hline 12 & SGC & -0.26 & -0.40 & SGC & -0.30 & -0.34 \\
\hline 13 & $\mathrm{HC}$ & 0.49 & 0.42 & $\mathrm{HC}$ & 0.38 & 0.43 \\
\hline 14 & $\mathrm{HAC}$ & 0.00 & 0.00 & HAC & 0.11 & 0.00 \\
\hline 15 & $\mathrm{HB} 2 \mathrm{C}$ & 0.16 & 0.09 & $\mathrm{HB} 2 \mathrm{C}$ & 0.19 & 0.16 \\
\hline 16 & HB3C & 0.14 & 0.09 & HB3C & 0.16 & 0.09 \\
\hline 17 & NY & -0.42 & -0.41 & NY & -0.49 & -0.39 \\
\hline 18 & CAY & 0.00 & -0.09 & CAY & -0.02 & -0.11 \\
\hline 19 & HY & 0.42 & 0.41 & HY & 0.37 & 0.39 \\
\hline 20 & HA1Y & 0.01 & 0.03 & HA1Y & 0.12 & 0.04 \\
\hline 21 & HA2Y & 0.01 & 0.03 & HA2Y & 0.06 & 0.04 \\
\hline 22 & HA3Y & -0.02 & 0.03 & HA3Y & 0.01 & 0.03 \\
\hline 23 & PA & 1.13 & 0.91 & PA & 1.08 & 0.90 \\
\hline 24 & O1A & -0.73 & -0.69 & O1A & -0.79 & -0.73 \\
\hline 25 & $\mathrm{O} 2 \mathrm{~A}$ & -0.74 & -0.69 & $\mathrm{O} 2 \mathrm{~A}$ & -0.80 & -0.66 \\
\hline 26 & O5B & -0.41 & -0.37 & O5B & -0.44 & -0.38 \\
\hline 27 & C5B & 0.00 & 0.00 & C5B & 0.04 & 0.00 \\
\hline 28 & C4B & 0.16 & 0.16 & C4B & 0.17 & 0.12 \\
\hline 29 & O4B & -0.61 & -0.57 & O4B & -0.57 & -0.56 \\
\hline 30 & C3B & 0.18 & 0.15 & $\mathrm{C} 3 \mathrm{~B}$ & 0.19 & 0.18 \\
\hline 31 & O3B & -0.77 & -0.73 & O3B & -0.72 & -0.75 \\
\hline 32 & $\mathrm{C} 2 \mathrm{~B}$ & 0.00 & -0.07 & $\mathrm{C} 2 \mathrm{~B}$ & 0.00 & -0.08 \\
\hline 33 & $\mathrm{O} 2 \mathrm{~B}$ & -0.75 & -0.71 & $\mathrm{O} 2 \mathrm{~B}$ & -0.71 & -0.72 \\
\hline 34 & C1B & 0.57 & 0.47 & C1B & 0.61 & 0.55 \\
\hline 35 & N9A & -0.29 & -0.21 & N9A & -0.31 & -0.23 \\
\hline 36 & $\mathrm{C} 8 \mathrm{~A}$ & 0.27 & 0.19 & C8A & 0.25 & 0.19 \\
\hline 37 & N7A & -0.66 & -0.58 & N7A & -0.68 & -0.59 \\
\hline 38 & $\mathrm{C} 5 \mathrm{~A}$ & 0.09 & 0.01 & $\mathrm{C} 5 \mathrm{~A}$ & 0.09 & 0.02 \\
\hline 39 & C6A & 0.75 & 0.76 & C6A & 0.72 & 0.75 \\
\hline 40 & N6A & -0.84 & -0.86 & N6A & -0.88 & -0.86 \\
\hline 41 & $\mathrm{~N} 1 \mathrm{~A}$ & -0.83 & -0.79 & N1A & -0.87 & -0.76 \\
\hline 42 & $\mathrm{C} 2 \mathrm{~A}$ & 0.67 & 0.58 & $\mathrm{C} 2 \mathrm{~A}$ & 0.65 & 0.55 \\
\hline 43 & $\mathrm{~N} 3 \mathrm{~A}$ & -0.86 & -0.82 & N3A & -0.90 & -0.80 \\
\hline 44 & $\mathrm{C} 4 \mathrm{~A}$ & 0.62 & 0.59 & $\mathrm{C} 4 \mathrm{~A}$ & 0.60 & 0.58 \\
\hline 45 & N1 & -0.84 & -0.91 & N1 & -0.82 & -0.79 \\
\hline 46 & $\mathrm{C} 2$ & 0.93 & 0.97 & $\mathrm{C} 2$ & 0.95 & 0.94 \\
\hline 47 & $\mathrm{O} 2$ & -0.77 & -0.81 & $\mathrm{O} 2$ & -0.68 & -0.70 \\
\hline 48 & N3 & -0.82 & -0.74 & N3 & -0.76 & -0.63 \\
\hline
\end{tabular}




\begin{tabular}{|c|c|c|c|c|c|c|}
\hline 49 & $\mathrm{C} 4$ & 0.71 & 0.82 & $\mathrm{C} 4$ & 0.73 & 0.66 \\
\hline 50 & $\mathrm{O} 4$ & -0.76 & -0.76 & $\mathrm{O} 4$ & -0.63 & -0.63 \\
\hline 51 & $\mathrm{C} 4 \mathrm{X}$ & -0.02 & -0.50 & C4X & 0.20 & 0.23 \\
\hline 52 & N5 & -0.79 & -0.35 & N5 & -0.62 & -0.70 \\
\hline 53 & C5X & 0.50 & 0.32 & C5X & 0.68 & 0.87 \\
\hline 54 & C6 & -0.46 & -0.55 & C6 & -0.50 & -0.53 \\
\hline 55 & $\mathrm{C} 7$ & 0.40 & 0.39 & $\mathrm{C} 7$ & 0.49 & 0.45 \\
\hline 56 & C7M & -0.52 & -0.36 & C7M & -0.58 & -0.40 \\
\hline 57 & C8 & -0.34 & -0.27 & C8 & -0.29 & -0.18 \\
\hline 58 & $\mathrm{C} 8 \mathrm{M}$ & 0.00 & 0.18 & $\mathrm{C} 8 \mathrm{M}$ & -0.02 & 0.01 \\
\hline 59 & C9 & 0.09 & -0.12 & C9 & 0.16 & 0.07 \\
\hline 60 & C9A & -0.37 & -0.12 & C9A & -0.36 & -0.47 \\
\hline 61 & N10 & 0.27 & -0.07 & N10 & 0.23 & 0.33 \\
\hline 62 & $\mathrm{C} 10$ & 0.19 & 0.59 & $\mathrm{C} 10$ & 0.36 & 0.29 \\
\hline 63 & $\mathrm{C} 1^{\prime}$ & -0.27 & -0.20 & $\mathrm{C} 1^{\prime}$ & -0.20 & -0.28 \\
\hline 64 & $\mathrm{C} 2^{\prime}$ & 0.19 & 0.21 & $\mathrm{C} 2^{\prime}$ & 0.13 & 0.14 \\
\hline 65 & $\mathrm{O} 2^{\prime}$ & -0.70 & -0.70 & $\mathrm{O} 2^{\prime}$ & -0.71 & -0.66 \\
\hline 66 & C3' & 0.26 & 0.10 & $\mathrm{C} 3^{\prime}$ & 0.27 & 0.13 \\
\hline 67 & O3' & -0.74 & -0.67 & O3' & -0.69 & -0.68 \\
\hline 68 & C4' & 0.18 & 0.02 & C4' & 0.19 & 0.10 \\
\hline 69 & O4' & -0.67 & -0.60 & O4' & -0.64 & -0.64 \\
\hline 70 & $\mathrm{C} 5^{\prime}$ & 0.00 & 0.00 & $\mathrm{C} 5^{\prime}$ & 0.12 & 0.00 \\
\hline 71 & O5' & -0.43 & -0.35 & O5' & -0.46 & -0.35 \\
\hline 72 & $\mathrm{P}$ & 1.07 & 0.91 & $\mathrm{P}$ & 0.99 & 0.92 \\
\hline 73 & O1P & -0.73 & -0.72 & O1P & -0.79 & -0.70 \\
\hline 74 & $\mathrm{O} 2 \mathrm{P}$ & -0.71 & -0.72 & $\mathrm{O} 2 \mathrm{P}$ & -0.77 & -0.73 \\
\hline 75 & O3P & -0.45 & -0.28 & O3P & -0.48 & -0.27 \\
\hline 76 & H5B2 & 0.00 & 0.00 & H5B2 & 0.12 & 0.00 \\
\hline 77 & H5B3 & 0.00 & 0.00 & H5B3 & 0.06 & 0.00 \\
\hline 78 & H4B & 0.10 & 0.11 & H4B & 0.11 & 0.11 \\
\hline 79 & Н3B & 0.08 & 0.12 & Н3B & 0.08 & 0.10 \\
\hline 80 & $\mathrm{H} 2 \mathrm{~B}$ & 0.10 & 0.13 & $\mathrm{H} 2 \mathrm{~B}$ & 0.10 & 0.13 \\
\hline 81 & H1B & 0.06 & 0.07 & H1B & 0.06 & 0.05 \\
\hline 82 & H8A & 0.15 & 0.18 & H8A & 0.15 & 0.19 \\
\hline 83 & $\mathrm{H} 2 \mathrm{~A}$ & 0.04 & 0.09 & $\mathrm{H} 2 \mathrm{~A}$ & 0.04 & 0.09 \\
\hline 84 & H3 & 0.41 & 0.41 & H3 & 0.42 & 0.41 \\
\hline 85 & H6 & 0.18 & 0.21 & H6 & 0.21 & 0.22 \\
\hline 86 & H8M2 & 0.18 & 0.11 & H8M2 & 0.17 & 0.20 \\
\hline 87 & H8M3 & 0.08 & 0.11 & H8M3 & 0.07 & 0.13 \\
\hline 88 & H9 & 0.13 & 0.18 & H9 & 0.12 & 0.16 \\
\hline 89 & H1'1 & 0.16 & 0.10 & H1'1 & 0.14 & 0.14 \\
\hline 90 & H1'2 & 0.11 & 0.10 & H1'2 & 0.11 & 0.14 \\
\hline 91 & $\mathrm{H} 2^{\prime}$ & 0.05 & 0.08 & $\mathrm{H} 2^{\prime}$ & 0.07 & 0.10 \\
\hline 92 & H3' & 0.08 & 0.15 & H3' & 0.09 & 0.14 \\
\hline 93 & H4' & 0.06 & 0.12 & H4' & 0.06 & 0.10 \\
\hline 94 & H5'1 & 0.00 & 0.00 & H5'1 & 0.05 & 0.00 \\
\hline 95 & $\mathrm{H} 5{ }^{\prime} 2$ & 0.00 & 0.00 & $\mathrm{H} 5{ }^{\prime} 2$ & 0.06 & 0.00 \\
\hline 96 & $\mathrm{HO}_{2}^{\prime}$ & 0.46 & 0.41 & $\mathrm{HO}_{2}^{\prime}$ & 0.45 & 0.42 \\
\hline 97 & H6A1 & 0.44 & 0.43 & H6A1 & 0.42 & 0.42 \\
\hline 98 & H6A2 & 0.45 & 0.43 & H6A2 & 0.44 & 0.45 \\
\hline 99 & HO4' & 0.43 & 0.46 & HO4' & 0.44 & 0.44 \\
\hline 100 & HO3' & 0.40 & 0.42 & HO3' & 0.43 & 0.41 \\
\hline 101 & $\mathrm{HO} 3 \mathrm{~B}$ & 0.43 & 0.43 & HO3B & 0.46 & 0.43 \\
\hline
\end{tabular}




\begin{tabular}{|l|lll|lll|}
102 & HO2B & 0.45 & 0.44 & HO2B & 0.48 & 0.44 \\
103 & H7M1 & 0.18 & 0.12 & H7M1 & 0.17 & 0.14 \\
104 & H7M2 & 0.17 & 0.12 & H7M2 & 0.17 & 0.11 \\
105 & H7M3 & 0.17 & 0.12 & H7M3 & 0.16 & 0.15 \\
106 & & & & HN5 & $\mathbf{0 . 3 6}$ & $\mathbf{0 . 3 1}$
\end{tabular}

Tabel S6. Lumiflavin (LFN) charges. (The transferring hydrogen and the accepting N5 atom of the cofactor are indicated in shading.)

\begin{tabular}{|c|c|c|c|c|c|c|}
\hline \multirow{2}{*}{$\begin{array}{c}\text { LFN } \\
\text { Atom ID }\end{array}$} & \multicolumn{3}{|c|}{ Reactant State } & \multicolumn{3}{|c|}{ Product State } \\
\hline & Atom Name & Initial (0 ps) & Final (60 ps) & Atom Name & Initial (0 ps) & Final (60 ps) \\
\hline 1 & N1 & -0.89 & -0.92 & $\mathrm{~N} 1$ & -0.80 & -0.78 \\
\hline 2 & $\mathrm{C} 2$ & 0.96 & 0.94 & $\mathrm{C} 2$ & 0.97 & 0.93 \\
\hline 3 & $\mathrm{O} 2$ & -0.79 & -0.81 & $\mathrm{O} 2$ & -0.68 & -0.69 \\
\hline 4 & N3 & -0.81 & -0.72 & N3 & -0.73 & -0.62 \\
\hline 5 & $\mathrm{C} 4$ & 0.73 & 0.77 & $\mathrm{C} 4$ & 0.71 & 0.67 \\
\hline 6 & $\mathrm{O} 4$ & -0.73 & -0.78 & $\mathrm{O} 4$ & -0.62 & -0.63 \\
\hline 7 & $\mathrm{C} 4 \mathrm{X}$ & -0.26 & -0.44 & $\mathrm{C} 4 \mathrm{X}$ & 0.26 & 0.21 \\
\hline 8 & N5 & -0.70 & -0.38 & N5 & -0.70 & -0.67 \\
\hline 9 & C5X & 0.43 & 0.19 & $\mathrm{C} 5 \mathrm{X}$ & 0.71 & 0.78 \\
\hline 10 & $\mathrm{C} 6$ & -0.53 & -0.47 & $\mathrm{C} 6$ & -0.47 & -0.46 \\
\hline 11 & $\mathrm{C} 7$ & 0.18 & 0.26 & $\mathrm{C} 7$ & 0.26 & 0.36 \\
\hline 12 & $\mathrm{C} 7 \mathrm{M}$ & -0.30 & -0.43 & $\mathrm{C} 7 \mathrm{M}$ & -0.45 & -0.36 \\
\hline 13 & C8 & 0.04 & 0.14 & C8 & 0.14 & -0.11 \\
\hline 14 & $\mathrm{C} 8 \mathrm{M}$ & -0.29 & -0.41 & $\mathrm{C} 8 \mathrm{M}$ & -0.47 & -0.36 \\
\hline 15 & C9 & -0.30 & -0.31 & C9 & -0.14 & -0.01 \\
\hline 16 & C9A & -0.05 & -0.12 & C9A & -0.35 & -0.36 \\
\hline 17 & N10 & -0.07 & 0.09 & N10 & 0.28 & 0.27 \\
\hline 18 & $\mathrm{C} 10$ & 0.56 & 0.48 & C10 & 0.34 & 0.31 \\
\hline 19 & $\mathrm{C} 1^{\prime}$ & -0.25 & -0.32 & $\mathrm{C} 1^{\prime}$ & -0.26 & -0.24 \\
\hline 20 & $\mathrm{H} 3$ & 0.40 & 0.38 & $\mathrm{H} 3$ & 0.40 & 0.41 \\
\hline 21 & H6 & 0.22 & 0.20 & H6 & 0.20 & 0.21 \\
\hline 22 & H9 & 0.19 & 0.18 & H9 & 0.16 & 0.18 \\
\hline 23 & H7M1 & 0.09 & 0.15 & H7M1 & 0.14 & 0.12 \\
\hline 24 & H7M2 & 0.09 & 0.14 & H7M2 & 0.14 & 0.12 \\
\hline 25 & H7M3 & 0.09 & 0.14 & H7M3 & 0.14 & 0.12 \\
\hline 26 & H8M1 & 0.09 & 0.13 & H8M1 & 0.15 & 0.12 \\
\hline 27 & H8M2 & 0.09 & 0.14 & H8M2 & 0.14 & 0.12 \\
\hline 28 & H8M3 & 0.09 & 0.14 & H8M3 & 0.15 & 0.12 \\
\hline 29 & H1'1 & 0.11 & 0.11 & H1'1 & 0.13 & 0.08 \\
\hline 30 & H1'2 & 0.11 & 0.10 & H1'2 & 0.13 & 0.08 \\
\hline 31 & H1'3 & 0.11 & 0.11 & H1'3 & 0.12 & 0.08 \\
\hline 32 & & & & HN5 & 0.40 & 0.32 \\
\hline
\end{tabular}




\section{MD parameters for the non-standard residues in the EVB region as defined in the}

\section{MOLARIS program package}

The bond energy between atoms in the EVB regions is defined with the Morse potential:

$$
E_{\mathrm{b}}=D\left(1-e^{-\beta\left(r-r_{0}\right)}\right)
$$

$D$ is equal to the square root of the product of Morse coefficients $\left(M_{\mathrm{A}}\right.$ and $\left.M_{\mathrm{B}}\right)$, where $\mathrm{A}$ and $\mathrm{B}$ are the bond atom types. $r_{0}$ is calculated by summing the Morse radii of the specific atom types $\left(r_{0}=r_{\mathrm{A}}+r_{\mathrm{B}}\right)$, while $\beta$ is equal to $2.0 \AA^{-1}$.

The equilibrium angle is calculated with a harmonic approximation of the following form:

$$
E_{\text {angle }}=F\left(\varnothing-\emptyset_{0}\right)^{2}
$$

For the calculations of nonbonding interactions MOLARIS uses the sum of an exponential term and the 6-12 van der Waals energy contributions:

$$
E_{\text {nonbond }}=\sqrt{C_{A} C_{B}} \cdot e^{-\sqrt{\beta_{A} \beta_{B}} r}+k_{A}^{A} \cdot k_{B}^{A} \cdot r^{-12}+k_{A}^{B} \cdot k_{B}^{B} \cdot r^{-6}
$$

For bonded EVB atoms, only the exponential term is considered and the van der Waals coefficients are set to zero (i.e. $k_{\mathrm{X}}^{A}=0$ and $k_{\mathrm{X}}^{B}=0$ ). If the atoms are not bonded in any state, the exponential term is set to zero (i.e. $C_{\mathrm{X}}=0$ ). The coefficients for atom types present in the EVB region are summarized in the table below. 
Table S7. Bonded parameters for the different atom types in the EVB region.

\begin{tabular}{ccccccccc}
\hline Atom type & $\boldsymbol{M}_{\mathbf{X}}[\mathbf{k c a l}]$ & $\boldsymbol{r}_{\mathbf{X}}[\AA ⿻$ & $\boldsymbol{F}\left[\mathbf{k c a l} /\left(^{(}\right)^{2}\right]$ & $\boldsymbol{\phi}_{\mathbf{0}}\left[^{\circ}\right]$ & $\boldsymbol{C}_{\mathbf{X}}[\mathbf{k c a l}]$ & $\boldsymbol{\beta}_{\mathbf{X}}\left[\AA^{-1}\right]$ & $\boldsymbol{k}_{\mathbf{X}}^{\mathbf{A}}\left[\mathbf{k c a l} \AA^{12}\right]$ & $\boldsymbol{k}_{\mathbf{X}}^{\mathbf{B}}\left[\mathbf{k c a l} \AA^{6}\right]$ \\
\hline $\mathrm{H} 0$ & 105.0 & 0.4 & 0.0 & 0.0 & 5.0 & 2.5 & 7.00 & 0.0 \\
$\mathrm{~N}+$ & 92.0 & 0.7 & 50.0 & 120.0 & 60.0 & 2.5 & 774.0 & 24.0 \\
$\mathrm{~N} 0$ & 92.0 & 0.7 & 50.0 & 109.5 & 43.0 & 2.5 & 774.0 & 24.0 \\
$\mathrm{C} 0$ & 96.0 & 0.7 & 50.0 & 109.5 & 91.0 & 2.5 & 632.0 & 24.0 \\
$\mathrm{C}+$ & 96.0 & 0.7 & 50.0 & 120.0 & 91.0 & 2.5 & 632.0 & 24.0 \\
$\mathrm{O}-$ & 90.0 & 0.7 & 50.0 & 120.0 & 90.0 & 2.5 & 1400.0 & 24.0 \\
\hline
\end{tabular}

Atom types are defined according to their hybridization in the system, i.e. an $\mathrm{sp}^{3}$ hybridized $\mathrm{C}$ atom is defined as $\mathrm{C} 0$ type and an $\mathrm{sp}^{2}$ hybridized $\mathrm{C}$ atom as $\mathrm{C}+$ type. The rest of the system was parameterized with the ENZYMIX force field described by Lee and co-workers. ${ }^{8}$ 

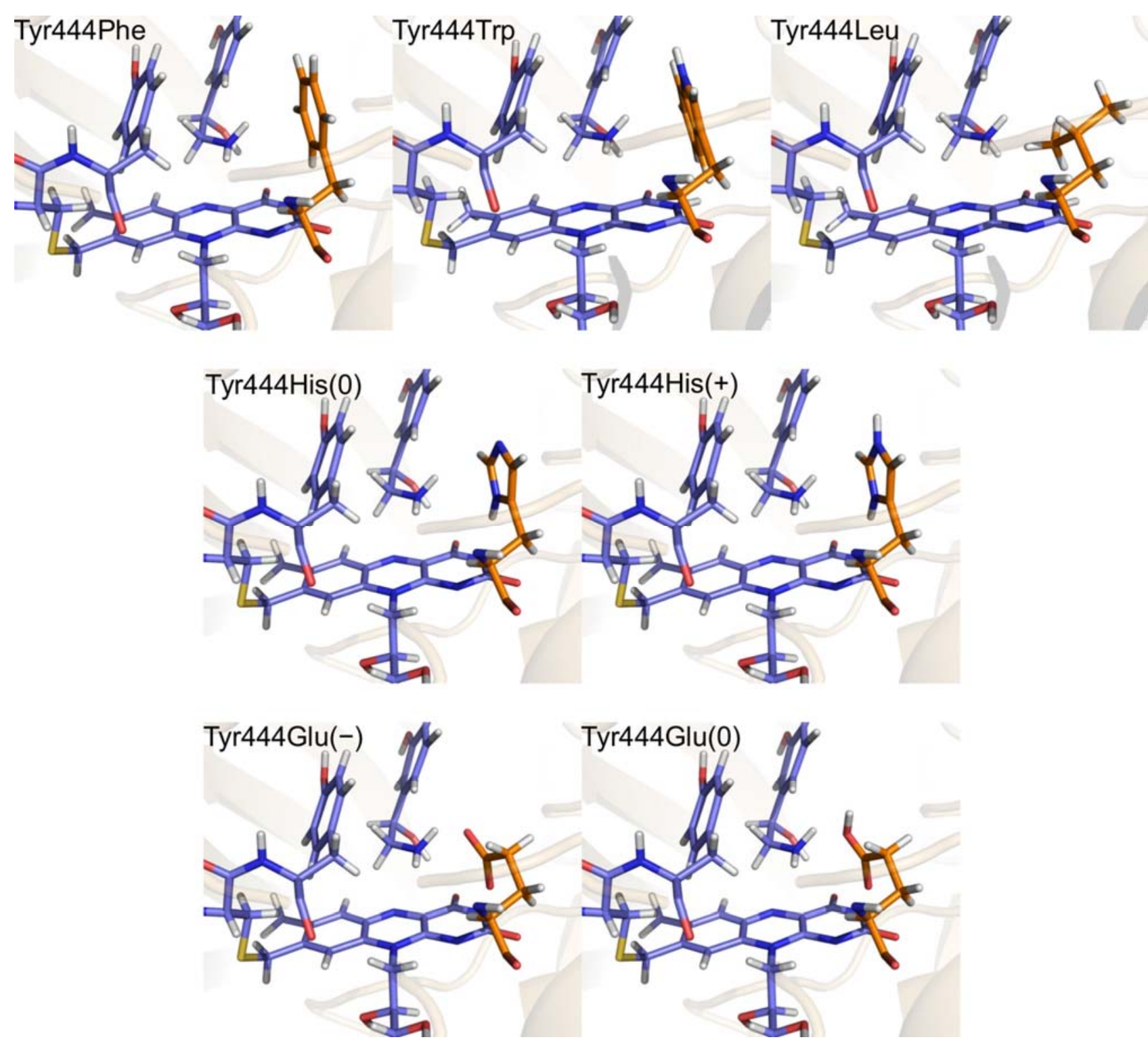

Figure S1. Initial placement of substrate in the studied mutated enzymes. The mutations are indicated by the orange color of the carbon atoms of the corresponding residues. 


\section{References}

1 O'Carroll, A. M.; Bardsley, M. E.; Tipton, K. F. The Oxidation of Adrenaline and Noradrenaline by the Two Forms of Monoamine Oxidase from Human and Rat Brain. Neurochem. Int. 1986, 8, 493500.

2 Li, M.; Hubálek, F.; Newton-Vinson, P.; Edmondson, D. E. High-Level Expression of Human Liver Monoamine Oxidase A in Pichia Pastoris: Comparison with the Enzyme Expressed in Saccharomyces Cerevisiae. Protein Expr. Purif. 2002, 24, 152-162.

3 Nandigama, R. K.; Edmondson, D. E. Structure-Activity Relations in the Oxidation of Phenethylamine Analogues by Recombinant Human Liver Monoamine Oxidase A. Biochemistry 2000, 39, 15258-15265.

4 Vintém, A. P. B.; Price, N. T.; Silverman, R. B.; Ramsay, R. R. Mutation of Surface Cysteine 374 to Alanine in Monoamine Oxidase A Alters Substrate Turnover and Inactivation by Cyclopropylamines. Bioorg. Med. Chem. 2005, 13, 3487-3495.

5 Wang, J.; Edmondson, D. E. Do Monomeric vs. Dimeric Forms of MAO-A Make a Difference? A Direct Comparison of the Catalytic Properties of Rat and Human MAO-A's. J. Neural Transm. 2007, 114, 721-724.

6 Cruz, F.; Edmondson, D. E. Kinetic Properties of Recombinant MAO-A on Incorporation into Phospholipid Nanodisks. J. Neural Transm. 2007, 114, 699-702.

7 Edmondson, D. E.; Binda, C.; Wang, J.; Upadhyay, A. K.; Mattevi, A. Molecular and Mechanistic Properties of the Membrane-Bound Mitochondrial Monoamine Oxidases. Biochemistry 2009, 48, 4220-4230. 
8 Lee, F. S.; Chu, Z. T.; Warshel, A. Microscopic and Semimicroscopic Calculations of Electrostatic Energies in Proteins by the POLARIS and ENZYMIX Programs. J. Comput. Chem. 1993, 14, 161185. 